\title{
Probabilistic Semi-Supervised Multi-Modal Hashing
}

Behnam Gholami

bb510@cs.rutgers.edu

Abolfazl Hajisami

hajisamik@cac.rutgers.edu
Computer Science Department
Rutgers university

Department of Electrical and Computer

Engineering

Rutgers university
In this paper, we propose a non-parametric Bayesian framework for multi-modal hash learning that takes into account the distance supervision (similarity/dissimilarity constraints). Our model embeds data of arbitrary modalities into a single latent binary feature with the ability to learn the dimensionality of the binary feature using the data itself. Given supervisory information (labeled similar and dissimilar pairs), we propose a novel discriminative term and develop a new Variational Bayes (VB) algorithm which incorporates that term into the proposed Bayesian framework.

Let $\boldsymbol{T}=[\boldsymbol{X}, \boldsymbol{Y}]$ be the observed bi-modal data matrix where $\boldsymbol{X}=\left[x_{1}, x_{2}, \ldots, x_{d}\right]_{M \times d}$ and $\boldsymbol{Y}=\left[y_{1}, y_{2}, \ldots, y_{d}\right]_{N \times d}$ denote the first modal and the second modal data matrix respectively, and $\boldsymbol{Z}=\left[z_{1}, z_{2}, \ldots, z_{d}\right]_{K \times d}$ denotes the latent binary code matrix.

In our VB framework, we truncate the length of the binary codes $(K)$ and we set it to a finite but large number. If $K$ is large enough, the analyzed multi-modal data using this number of bits, will reveal less than $K$ bits. In order to incorporate the information of the similarity/dissimilarity constraints into the VB algorithm, we first define a regularizer for the binary code $z_{i}$ as

$$
\begin{aligned}
\alpha\left(z_{i}\right)= & \frac{1}{\left|\mathcal{D}_{i}\right|} \sum_{j:(i, j) \in \mathcal{D}} K L\left(q_{z_{i}}\left(z_{i}\right) \| q_{z_{j}}\left(z_{j}\right)\right) \\
& -\frac{1}{\left|\mathcal{S}_{i}\right|} \sum_{j:(i, j) \in \mathcal{S}} K L\left(q_{z_{i}}\left(z_{i}\right) \| q_{z_{j}}\left(z_{j}\right)\right)
\end{aligned}
$$

where $K L(p \| q)$ denotes the KL divergence between two distributions $p$ and $q$, and $\mathcal{S}(\mathcal{D})$ denotes the set of similar (dissimilar) pairwise constraints. Intuitively, for each binary code $z, \alpha(z)$ should be large such that it best agrees with those constraints.

By defining the regularizer $\Omega(\boldsymbol{Z})=$ $\sum_{i=1}^{d} \alpha\left(z_{i}\right)$ for the binary code matrix $\boldsymbol{Z}$ using the set of similar/dissimilar pairs, we add this
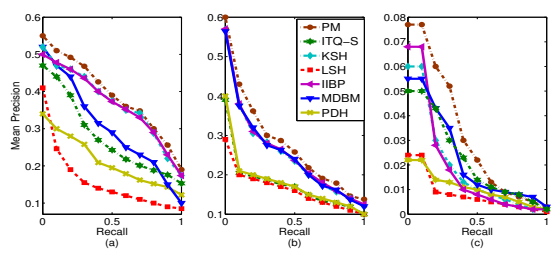

Figure 1: The result of category retrieval for image-to-image queries. (a) PASCAL-Sentence Dataset; (b) SUN Dataset (Euclidean ground truth computed from visual data); (c) SUN Dataset (Class label ground truth)

regularizer to the objective function of $\mathrm{VB}$ and solve the new optimization problem using the Coordinate Descent method.

We evaluate the proposed method on two benchmark bi-modal datasets: (1) The PASCAL-Sentence 2008 dataset [1] consists of 1000 images categorized into 20 classes. (2) The SUN-Attribute dataset [2] contains 102 attribute labels for each of the 14340 images from 717 categories. We compare the performance of the proposed method against five state-of-the-art hashing methods (Fig. 1) using precision-recall curve as an accuracy measure. As can be seen, the proposed method outperforms the other state of the art (multi-modal) hashing methods.

[1] Ali Farhadi, Mohsen Hejrati, Mohammad Amin Sadeghi, Peter Young, Cyrus Rashtchian, Julia Hockenmaier, and David Forsyth. Every picture tells a story: Generating sentences from images. In Computer Vision-ECCV 2010, pages 15-29. Springer, 2010.

[2] Genevieve Patterson and James Hays. Sun attribute database: Discovering, annotating, and recognizing scene attributes. In $C V P R$, pages 2751-2758. IEEE, 2012. 